\title{
Population growth, age structure, and age-specific productivity
}

\section{Does a uniform age distribution minimize lifetime wages?}

\author{
David Lam* \\ Department of Economics and Population Studies Center, University of Michigan, \\ Ann Arbor, MI 48109, USA
}

Received March 3, 1989 / Accepted August 1, 1989

\begin{abstract}
Motivated by empirical evidence that fluctuations in age structure affect relative wages across age groups, this paper asks whether there is a steady-state age distribution that maximizes the lifetime wages of a representative worker. The paper proves the surprising result that in a pure labor economy with any constant returns technology, a uniform age distribution minimizes lifetime wages. Skewed age distributions, generated by either positive or negative population growth rates, generate unambiguously higher lifetime wages than a stationary population, in spite of possible reductions in per capita output in every period. The presence of non-labor factors complicates, but does not necessarily reverse, this result. The paper relates the beneficial effects of higher rates of population growth on lifetime wages in a pure labor economy with imperfect substitutability across age groups to the benefits of population growth that appear in overlapping-generation consumption loan models with intergenerational transfers.
\end{abstract}

\section{Introduction}

Empirical research on the effects of cohort size on wages documents the sensitivity of age-specific wages to the relative sizes of age groups. The effects of cohort size on wages were first documented for the case of the United States baby boom by Easterlin (1978), Welch (1979), and Freeman (1979). Subsequent research, such as Stapleton and Young $(1984,1988)$, Berger $(1985,1989)$, Bloom et al. (1988), Connelly (1986), Dooley and Gottschalk (1984), and Falaris and Peters (1988), has provided a rich literature exploring the relationship between cohort size and

\footnotetext{
A previous version of this paper was presented at the Economic Demography Workshop at the 1988 meetings of the Population Association of America. Helpful comments from Mark Berger, Theodore Bergstrom, Ronald Lee, Hal Varian, and Robert Willis are acknowledged.

* Present address: IPEA/INPES; Av. Pres. Antonio Carlos, 51, andar 14; Rio de Janeiro, RJ 20020, Brazil.
} 
wages. Debate continues on the persistence of cohort size effects over the life cycle and on the nature of behavioral responses to cohort size in schooling and fertility. The fundamental fact that the relative size of age groups affects age-specific wages has now been firmly established, however.

Although empirical research has focussed on the effects of short-term fluctuations in cohort size, the results suggest that wages will be influenced by age structure in long-term demographic steady-states as well. Even if all other features of two economies are similar, for example, workers in a rapidly growing population with a young age distribution can be expected to face a significantly different wage profile than workers in a population with a low growth rate and a relatively uniform age distribution. Considerable attention has been given to the role of age structure in models analyzing the economic effects of population growth such as Arthur and McNicoll (1977, 1978), Lee (1980), Lee and Lapkoff (1988), and Willis (1982). In spite of the economic and demographic richness of the overlapping-generations models in this literature, however, the models have never incorporated effects of age structure on age-specific productivity. This paper attempts to fill this gap by analyzing the relationship between population growth rates and life cycle wage profiles, explicitly recognizing the possibility of imperfect substitutability of workers of different ages.

In previous research on the economic effects of population growth, two basic economic forces drive the results. The first is the effect of population growth on capital-labor ratios, typified by Solow's (1956) neoclassical growth model. Higher rates of population growth in such a model unambiguously lower steady state per capita income and consumption due to a capital dilution effect analogous to an increased rate of capital depreciation. The second common effect of population growth is an intergenerational transfer effect typified by Samuelson's (1958) original consumption loan model. In its simplest version, increased population growth leads to unambiguously higher per capita lifetime utility through what amounts to a perpetually underfunded pay-as-you-go social security system. A number of authors have attempted to combine these two effects into a single model, beginning with Samuelson (1975). Samuelson's initial attempt included an instructive error, with his first order conditions for an optimum growth rate implying a welfare minimum for a large class of production and utility functions. As pointed out by Deardorff (1976), when both production and utility are CobbDouglas, the benefits from increasing capital-labor ratios in Samuelson's model produce unbounded increases in lifetime utility as the population growth rate decreases from the critical point implied by Samuelson's first order conditions. At the same time, the benefits of intergenerational transfers in the model produce unbounded increases in lifetime utility as the growth rate increases from the critical point. No finite optimum population growth rate exists for this and other cases, a result explored in detail in Samuelson's (1976) reply to Deardorff. Models with more general treatments of age structure have been developed by Arthur and McNicoll (1977, 1978), Lee (1980), and Willis (1982). These models continue to be driven by the two forces of capital-dilution and intergenerational transfers, however, as the mechanism through which population growth and age structure affect economic welfare. None of these models has considered the effects of age structure on age-specific labor productivity, a potentially important alternative 
mechanism through which population growth and age structure will have direct economic effects.

Section 2 of this paper analyzes the relationship between factor proportions and marginal products for the simple case of a pure labor economy in which labor can be divided into two types. The section proves that for any concave constant returns production function, the sum of the two marginal products is minimized when there are equal numbers of the two types of workers. Section 3 illustrates the results for the case of a CES production function with two types of labor, and contrasts the effect of age structure on lifetime wages with the effect on total output per period. The implications of non-labor factors are considered in Sect. 4. Section 5 analyzes the effects of fertility-induced changes in population growth rates on age-specific wages in a stable population with any number of age groups. A uniform age distribution is shown to minimize steady state total lifetime wages in a pure labor economy with any constant returns technology. Effects of discounting the wage stream are considered in Sect. 6. Sections 7 and 8 incorporate effects of age structure on age-specific labor productivity into overlapping generations models of the effects of population growth on life cycle consumption profiles. Previous comparative steady state results on the effects of population growth on lifetime consumption are shown to generalize in a surprisingly straightforward way when the conventional assumption that workers of different ages are perfect substitutes is replaced with a completely general production function. Section 9 compares the results for this model with the implications of Samuelson's original consumption loan model.

\section{Factor proportions and factor payments under constant returns technology}

A fundamental but little recognized property of linearly homogeneous functions forms the foundation for the results developed below. The simplest form of the result can be demonstrated for an economy with two types of workers:

Proposition 1. If $L$ total workers are divided into two types, $L_{1}$ and $L_{2}$, and total output is given by a concave constant returns to scale production function $Y=F\left(L_{1}, L_{2}\right)$, the sum of the two marginal products $F_{1}+F_{2}$ attains a global minimum when $L_{1}=L_{2}$.

To prove the result, consider the problem of choosing the fraction $\pi_{1}$, where $L_{1}=\pi_{1} L$ and $L_{2}=\left(1-\pi_{1}\right) L$. Assuming that workers of each type are paid their marginal products, what effect will the choice of $\pi_{1}$ have on the sum of the two wages $W=w_{1}+w_{2}=F_{1}+F_{2}$ ? Noting that

$$
\frac{\partial L_{2}}{\partial \pi_{1}}=-\frac{\partial L_{1}}{\partial \pi_{1}}=-L
$$

it follows that

$$
\begin{aligned}
\frac{\partial W}{\partial \pi_{1}} & =\left(F_{11}+F_{21}\right) \frac{\partial L_{1}}{\partial \pi_{1}}+\left(F_{12}+F_{22}\right) \frac{\partial L_{2}}{\partial \pi_{1}} \\
& =\left(F_{11}+F_{21}-F_{12}-F_{22}\right) L=\left(F_{11}-F_{22}\right) L,
\end{aligned}
$$


where $F_{i j}=\partial^{2} F / \partial L_{i} \partial L_{j}$. The sign of $F_{11}-F_{22}$ will be indeterminate in general, but becomes a simple function of $\pi_{1}$ under constant returns to scale. Under constant returns it will be analytically convenient to normalize by the total number of workers. Using lower case letters to denote per worker quantities, define the per worker production function $y=f\left(L_{1}, L_{2}\right)=F\left(\pi_{1}, \pi_{2}\right)$, where $\pi_{i}=L_{i} / L$. The properties of homogeneous functions require that the derivatives of the total production function $F$ are related to the derivatives of the per worker production function $f$ by the conditions $F_{i}=f_{i}, F_{i j}=f_{i j} L^{-1}$, and $F_{i j k}=f_{i j k} L^{-2}$, where $F_{i}=\mathrm{\partial} F / \mathrm{\partial} L_{i}, f_{i}=\mathrm{\partial} f / \mathrm{\partial} \pi_{i}$, etc. The condition on first derivatives implies that wages are independent of total population size, while the condition on second derivatives simplies that (1) can be reduced to $\partial W / \partial \pi_{1}=f_{11}-f_{22}$, a result that is independent of $L$. Given constant returns, Euler's theorem implies that $f_{11} \pi_{1}+$ $f_{21} \pi_{2}=0$. Using the fact that $f_{21}=f_{12}$, this condition implies that

$$
f_{22}=f_{11}\left(\frac{\pi_{1}}{1-\pi_{1}}\right)^{2} \text {. }
$$

Substituting into (1), then

$$
\frac{\partial W}{\partial \pi_{1}}=f_{11}\left[1-\left(\frac{\pi_{1}}{1-\pi_{1}}\right)^{2}\right]=f_{11}\left[\frac{1-2 \pi_{1}}{\left(1-\pi_{1}\right)^{2}}\right] .
$$

By inspection, the derivative in (2) is equal to zero when $\pi_{1}=0.5$. To see whether this critical point is a minimum or maximum, differentiation of (2) gives

$$
\frac{\partial^{2} W}{\partial \pi_{1}^{2}}=\left(f_{111}-f_{112}\right)\left[\frac{1-2 \pi_{1}}{\left(1-\pi_{1}\right)^{2}}\right]-2 f_{11}\left(1-\pi_{1}\right)^{-2}+2 f_{11}\left[\frac{1-2 \pi_{1}}{\left(1-\pi_{1}\right)^{3}}\right] \text {. }
$$

The first and last terms equal zero when $\pi_{1}=0.5$, making the entire expression unanbiguously positive if $f_{11}<0$. Equal division of the workers therefore gives the global minimum lifetime wages for any concave production function. It is clear by inspection of (2) that the derivative is positive for all $\pi_{1}>0.5$, and is negative for all $\pi_{1}<0.5$ as long as $f_{11}<0$. This establishes Proposition 1. If $L_{1}$ and $L_{2}$ are the number of young and old workers respectively, then $\left(L_{1} / L_{2}\right)-1$ is the labor force growth rate. A worker passing through the labor force with one period in each age group will earn $W$ total lifetime wages. The result in (2), then, implies that for a pure labor economy with constant returns to scale, a stationary population (zero growth rate) produces the lifetime wage minimizing age structure. Either a positive or negative growth rate of the labor force will lead to greater lifetime wages for all workers. There is no finite growth rate that maximizes lifetime wages. As seen in (2), lifetime wages continually increase with increases in $\pi_{1}$ above 0.5 or with decreases in $\pi_{1}$ below 0.5 .

Although an economy without capital is an unrealistic abstraction, it is an important point of departure for more complete models, and follows in the spirit of Samuelson's (1958) original consumption-loan model. Since the introduction of imperfect labor substitutability across age groups significantly changes the relationship between population growth and wages in a Samuelson-type overlapping generations model, it is important to begin with this pure case in which workers of different ages are the only inputs, with their relative proportions com- 
pletely determining wages. An example of the effect driving Proposition 1 can be seen in Stapleton and Young's (1988) model of relative wages and cohort size, where a pure labor economy with two age groups and constant returns technology are assumed. Stapleton and Young observe that increasing the population growth rate from zero raises the wages of old workers more than it decreases the wages of young workers, an effect that is greater the lower the elasticity of substitution (equivalent to a "more concave" production function, i.e. a greater absolute value of $f_{11}$ in Eq. (2) above). The importance to the result in Proposition 1 of the assumption of only two age groups and the assumption that there are no productive inputs other than labor will be analyzed in detail below. It will be seen that the basic result is unaffected by generalizing to any arbitrary number of age groups. The result is not necessarily robust to the introduction of capital into the production function, however, although it will be shown that we can never rule out the case in which a uniform age distribution minimizes lifetime wages without imposing restrictions on the production function.

\section{An illustration with CES technology}

Proposition 1 holds for any concave constant returns production function in a pure labor economy, with no assumption about productivity differences across ages or the elasticity of substitution between the two types of workers (other than the standard restrictions implied by concavity of the production function). It is interesting to compare this result to the effect of the choice of $\pi_{1}$ on total output per period. Since $\partial Y / \partial \pi_{1}=F_{1}-F_{2}$, total output will be maximized when the marginal products of the two types or workers are equal. This could occur at any division of workers in general, with the result depending on the specific form of the production function. Consider, for example, a CES production function $Y=\left[\beta L_{1}^{o}+(1-\beta) L_{2}^{\rho}\right]^{1 / \varrho}$, which can be rewritten as

$$
Y=L\left[\beta \pi_{1}^{\varrho}+(1-\beta)\left(1-\pi_{1}\right)^{\varrho}\right]^{1 / \varrho} .
$$

Given $\varrho$ and $\beta$, there is a unique value of $\pi_{1}$, the proportion of the labor force in the younger age group, that equates the marginal products of the two ages of workers and therefore maximizes the value of total output. Since $F_{1}-F_{2}=$ $(Y / L)^{1-\varrho}\left[\beta \pi_{1}^{\varrho-1}-(1-\beta)\left(1-\pi_{1}\right)^{\varrho-1}\right]$, output per period attains a maximum when

$$
\frac{\pi_{1}}{1-\pi_{1}}=\left[\frac{\beta}{1-\beta}\right]^{\sigma},
$$

where $\sigma=1 /(1-\varrho)$. If the two types of workers have equal productivity parameters, i.e. $\beta=0.5$, then total output will always be maximized when $\pi_{1}=0.5$, implying a uniform age distribution. If $\beta \neq 0.5$ then the division of labor that maximizes total output will depend on the elasticity of substitution. Restristing $\sigma \geq 0$ for concavity, (5) implies that if $\beta<0.5$, implying a productivity advantage of older workers, the optimal $\pi_{1}$ will be less than 0.5 , since a greater proportion of older workers will be required to equate the marginal products of the two age groups. 


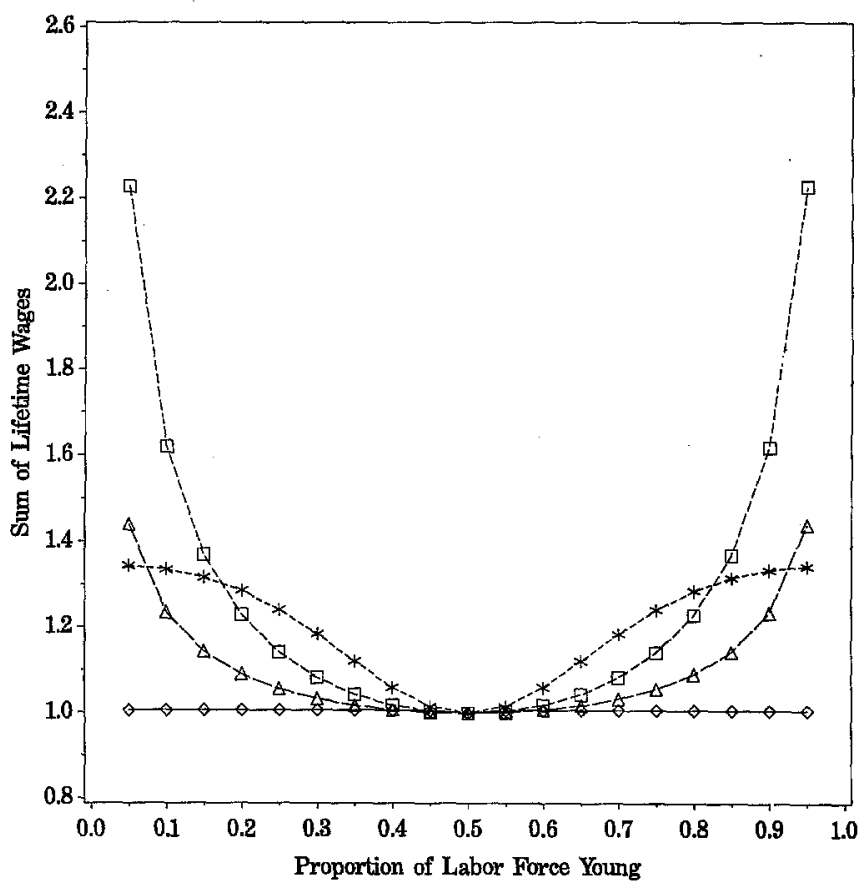

Fig. 1. Total lifetime wages as a function of proportion of labor force young. CES technology $Y=L\left[\beta \pi_{1}^{\varrho}+(1-\beta)\left(1-\pi_{1}\right)^{\varrho}\right]^{1 / \varrho}, \beta=0.5$. Alternative values of $\sigma=1 /(1-\varrho)$. $\sigma=\diamond \diamond \diamond 0.01$; $*-*-* 0.3 ; \square-\square-\square 1.1 ; \Delta-\triangle-\triangle 2.7$

Although the division of workers that maximizes total output depends on both $\sigma$ and $\beta$, Proposition 1 requires that for all values of $\beta$ and $\sigma$ total lifetime wages $w_{1}+w_{2}$ must attain a global minimum when there are equal numbers of workers of each type. The contrast between the effects of age structure on output and the effects of age structure on lifetime wages are shown graphically in Figs. $1-4$. Figure 1 shows the relationship between total lifetime wages, $w_{1}+w_{2}$ and the proportion of workers in the young age group for the case in which $\beta=0.5$ and for four alternative elasticities of substitution. As required by Proposition 1, lifetime wages attain a minimum at $\pi_{1}=0.5$ for all values of $\sigma$.

Figure 2 shows the relationship between the proportion of workers in the young age group and total output for the same production function. Given $\beta=0.5$, a uniform age distribution always maximizes total output for any value of $\sigma$. If the age distribution of workers remains constant and each worker spends one period as type 1 and one period as type 2, the example of Figs. 1-2 implies the paradoxical condition that the distribution of workers that maximizes total output in each period is the distribution that minimizes each worker's lifetime income. The apparent contradiction results from the fact that total (or per worker) output in each period need not have any relation to a representative worker's lifetime welfare.

The case in which there is a productivity difference between young and old workers is shown in Figs. 3 and 4. Continuing with the CES production function 


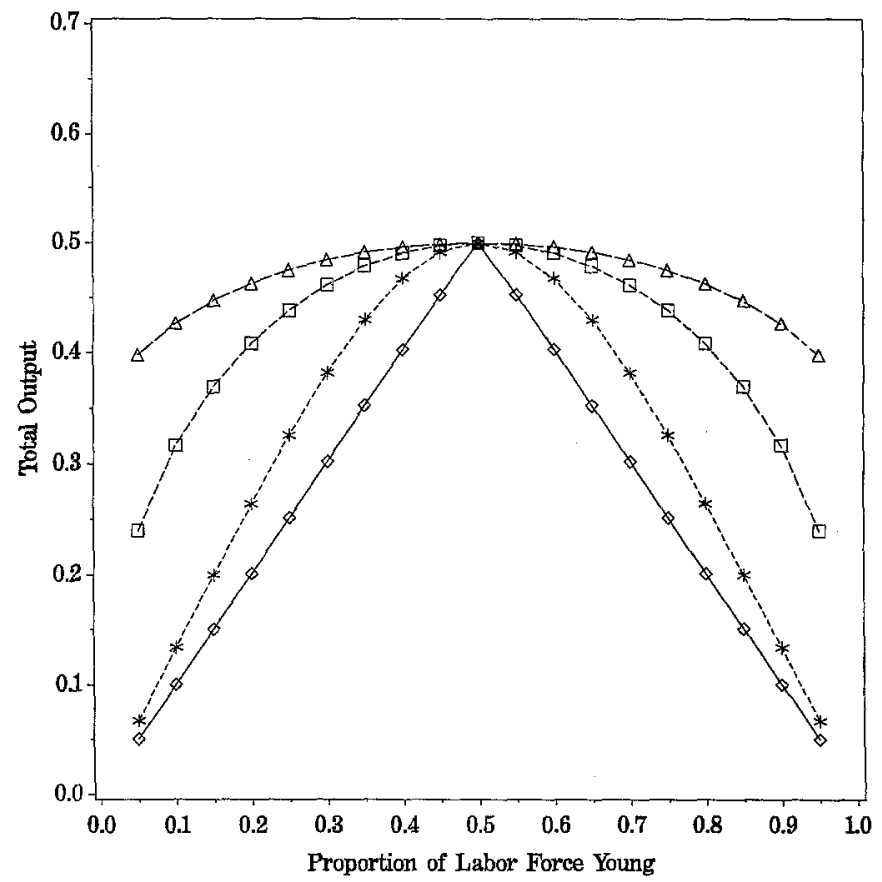

Fig. 2. Total output per period as a function of proportion of labor force young. CES technology $Y=L\left[\beta \pi_{1}^{\varrho}+(1-\beta)\left(1-\pi_{1}\right)^{\varrho}\right]^{1 / \varrho}, \beta=0.5$, Alternative values of $\sigma=1 /(1-\varrho) . \sigma=\diamond-\diamond \diamond 0.01$; $*-*-* 0.3 . \square-\square-\square 1.1 ; \triangle-\triangle-\triangle 2.7$

for two ages of workers in (4), Fig. 3 shows the relationship between total lifetime wages and the proportion of workers in the young age group for the case in which $\beta=0.3$ and for the same four alternative elasticities of substitution used in Fig. 1. Once again, as required by Proposition 1, lifetime wages attain a minimum at $\pi_{1}=0.5$ for all values of $\sigma$. The difference between this case and the equal productivity case of Fig. 1 is that the shape of the relationship between $w_{1}+w_{2}$ and $\pi_{1}$ is altered away from the critical point $\pi_{1}=0.5$.

Figure 4 shows the relationship between the proportion of workers in the young age group and total output for the same production function. It is no longer the case that $\pi_{1}=0.5$ maximizes total output, except in the limit as $\sigma \rightarrow 0$. The output-maximizing value of $\pi_{1}$ decreases with $\sigma$, since a higher ratio of older workers to young workers is required to equilibrate their marginal products as the degree of substitutability increases, given the productivity advantage of older workers $(\beta<0.5)$.

Figures 1-4 demonstrate that in the simple case in which workers seek to maximize lifetime income in a pure labor economy, a uniform age distribution is always the worst of all possible worlds, even though it may generate the highest per capita income in each period. It is important to note that even if a worker could choose the steady state age distribution in the population, maximizing lifetime wages would not necessarily be an appropriate objective function for the worker. The effect of age structure on lifetime wages provides an interesting base- 


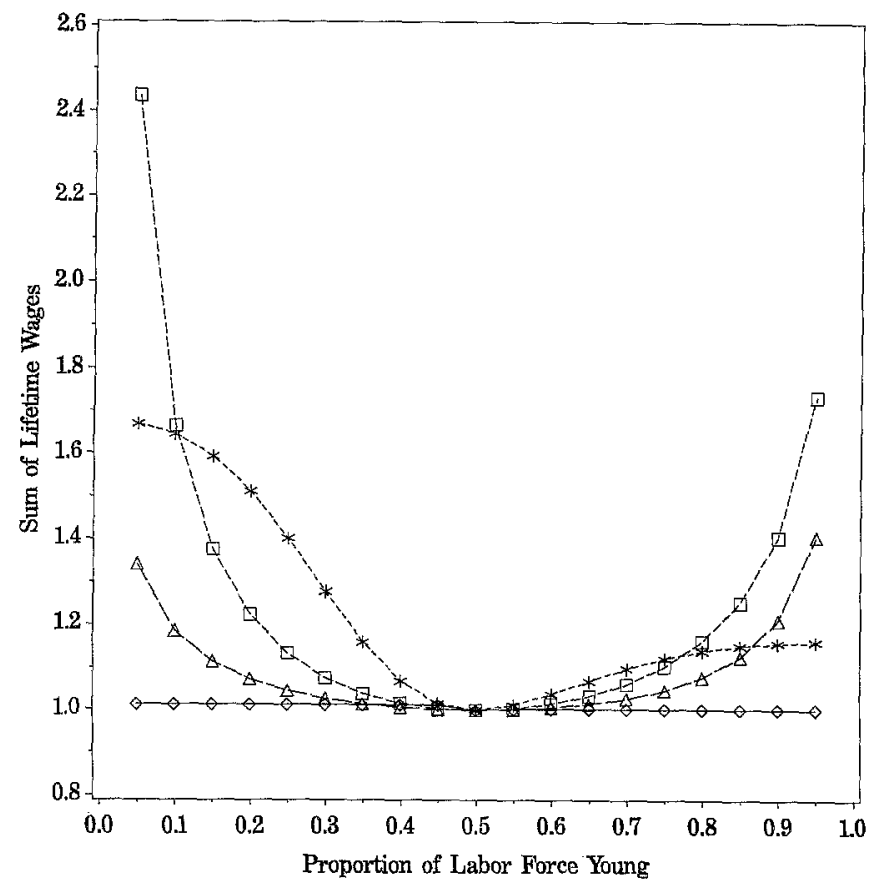

Fig. 3. Total lifetime wages as a function of proportion of labor force young. CES technology $Y=L\left[\beta \pi_{1}^{\varrho}+(1-\beta)\left(1-\pi_{1}\right)^{\varrho}\right]^{1 / \varrho}, \beta=0.3$. Alternative values of $\sigma=1 /(1-\varrho) . \sigma=\diamond \diamond \diamond 0.01$; $*-*-* 0.3 ; \square-\square-\square 1.1 ; \triangle-\triangle-\triangle 2.7$

line for the analysis, however. The effects of discounting lifetime wages are considered below, along with consideration of the effects of age structure on the possibilities for intertemporal consumption smoothing.

To see the implications of Proposition 1, consider a rapidly growing population, in which there are always many times more young workers than old workers. Young workers may have much lower wages than old workers, but Proposition 1 guarantees that $w_{1}+w_{2}$ is greater than it would be if the same number of workers were rearranged so that there were equal numbers at each age. The apparent inefficiency that total output in each period could be increased if there were equal numbers at each age is unimportant to the workers, as long as the positive growth rate can be sustained. If maximizing lifetime wages were an appropriate welfare criterion, any potential new worker would choose to enter the rapidly growing population rather than a stationary population as long as the growth rate persists throughout the worker's lifetime. The depressed ages earned while young are guaranteed to be more than offset by the higher wages earned while old. The requirement of a perpetual positive population growth rate recall the "intergenerational Ponzi scheme" nature of the gains to higher population growth rates in Samuelson's consumption-loan model. The effect of population growth in the presence of imperfect substitutability across age groups is conceptually quite different than the effect of population growth in Samuelson's model, however. Similarities and differences between the results derived here for the relationship 


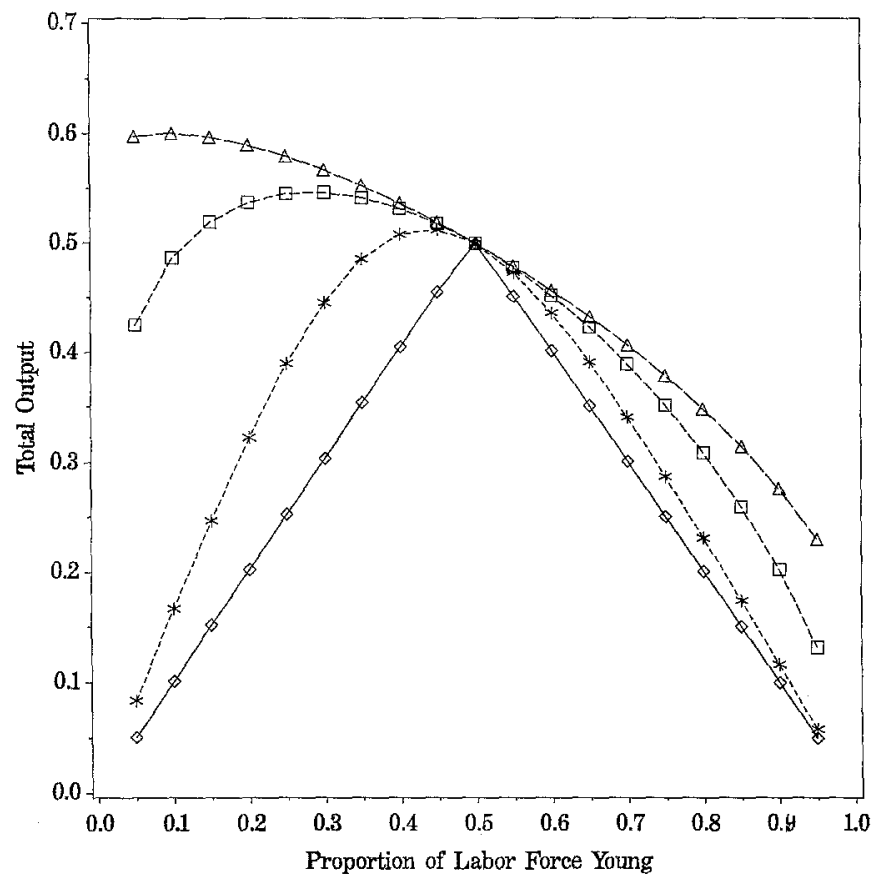

Fig. 4. Total output per period as a function of proportion of labor force young. CES technology $Y=L\left[\beta \pi_{1}^{\varrho}+(1-\beta)\left(1-\pi_{1}\right)^{\varrho}\right]^{1 / \varrho}, \beta=0.3$. Alternative values of $\sigma=1 /(1-\varrho) . \sigma=\diamond \diamond \diamond 0.01$; $*-*-* 0.3 ; \square-\square-\square 1.1 ; \triangle-\triangle-\triangle 2.7$

between age structure and lifetime wages and the role of age structure in Samuelson's pure consumption loan economy will be analyzed below.

\section{Effects of non-labor factors of production}

The result in Proposition 1 that a uniform age distribution minimizes lifetime wages is not necessarily robust to the introduction of non-labor factors of production. The effect of introducing a non-labor factor can be seen by considering an augmented production function, $Y=F\left(L_{1}, L_{2}, K\right)$, where the new factor $K$ can be thought of as a fixed resource, such as land, or as a reproducible factor, such as capital. (Distinctions between the two types of non-labor factors will be discussed below.) Assuming that $K$ is exogenous and unaffected by the choice of $\pi_{1}$, the introduction of $K$ has no effect on the derivation of (1), so under constant returns it is still true that $\mathrm{\partial} W / \partial \pi_{1}=\left(f_{11}-f_{22}\right)$. Euler's theorem now implies that $f_{11} \pi_{1}+f_{21} \pi_{2}=-f_{k 1} k$, where $k=K / L$, and therefore that

$$
f_{11}=-f_{k 1} \frac{k}{\pi_{1}}-f_{12} \frac{1-\pi_{1}}{\pi_{1}} .
$$

Using the analogous expression for $f_{22}$, and substituting into (1), the derivative in (2) generalizes to 


$$
\frac{\partial W}{\partial \pi_{1}}=f_{11}\left[\frac{1-2 \pi_{1}}{\left(1-\pi_{1}\right)^{2}}\right]+k\left[\frac{f_{k 2}}{\pi_{2}}-\frac{f_{k 1}}{\pi_{1}}\right] .
$$

The first term in (6) is zero when $\pi_{1}=0.5$, is positive for all $\pi_{1}>0.5$, and is negative for all $\pi_{1}<0.5$. The sign of the second term in (6) is indeterminate in general, and depends on the complementarity between $K$ and workers of different ages. Note, however, that if $W$ attains a critical point at $\pi_{1}=0.5$, then it must be the case that $f_{k 1}=f_{k 2}$ at that point, since the first term must equal zero. The second derivative is

$$
\begin{aligned}
\frac{\partial^{2} W}{\partial \pi_{1}^{2}}= & \left(f_{111}-f_{112}\right)\left[\frac{1-2 \pi_{1}}{\left(1-\pi_{1}\right)^{2}}\right]-2 f_{11}\left(1-\pi_{1}\right)^{-2}+2 f_{11}\left[\frac{1-2 \pi_{1}}{\left(1-\pi_{1}\right)^{3}}\right] \\
& +k\left[\pi_{2}^{-1}\left(f_{k 12}-f_{k 22}\right)-\pi_{2}^{-2} f_{k 2}+\pi_{1}^{-2} f_{k 1}-\pi_{1}^{-1}\left(f_{k 11}-f_{k 12}\right)\right] .
\end{aligned}
$$

If $W$ attains a critical point at $\pi_{1}=0.5$, implying that $\pi_{1}=\pi_{2}$ and $f_{k 1}=f_{k 2}$, then (7) reduces to

$$
\frac{\partial^{2} W}{\partial \pi_{1}^{2}}=\frac{-f_{11}}{4}+2 k\left(f_{k 12}-f_{k 11}+f_{k 12}-f_{k 22}\right) \text {. }
$$

The first term in (8) will always be positive under concavity. No general restrictions can be placed on the third derivatives in the second term in order to make the sign of the term unambiguous. We cannot rule out the possibility that the term is negative and large enough in absolute value to offset the positive first term. It is possible, then, that in the presence of other factors a uniform distribution of workers will maximize rather than minimize lifetime wages. It is also possible that a uniform distribution continues to minimize lifetime wages, however, as it does in the absence of non-labor factors. Some obvious restrictions on the third derivatives will guarantee that the second derivative is positive when $W\left(\pi_{1}\right)$ attains a critical point at $\pi_{1}=0.5$. The simplest is that $f_{k 12}=f_{k 11}=f_{k 22}$ when $\pi_{1}=\pi_{2}$ and $f_{k 1}=f_{k 2}$. This implies a symmetry in the relationship between $K$ and the two kinds of labor that is consistent with the requirement that $f_{k 1}=f_{k 2}$ when $\pi_{1}=\pi_{2}$. If the production function can be written as $F\left(K, G\left(L_{1}, L_{2}\right)\right)$, for example, then conditional on there being a critical point in $W\left(\pi_{1}\right)$ at $\pi_{1}=0.5$, that point will be a minimum if $G_{11}=G_{22}$ when $L_{1}=L_{2}$.

If $K$ represents reproducible capital, rather than a fixed resource like land, then there may be a direct effect of the age distribution on $K$. The effects of population growth and age structure on capital-labor ratios have been the principle focus of most models of the economic effects of population growth. Although a complete treatment of the relationship between population growth and capital accumulation is beyond the scope of this paper, the issue will be addressed below in analyzing golden rule steady states.

\section{Population growth, age structure, and lifetime wages}

A more complete model of the relationship between age structure and wage profiles can be constructed by considering a stable population with an arbitrary num- 
ber of age groups, a constant population growth rate, and a general production function defined over workers of different ages. The number of persons aged $i$ at time $t$, denoted $L_{i, t}$, is by definition $L_{i, t}=B_{t-i} p_{i}$, where $B_{t}$ denotes births in period $t$ and $p_{i}$ denotes the probability of survival from birth to age $i$, assumed to be invariant over time. If age-specific fertility and mortality rates remain constant over time, then by well known ergodicity properties (see Arthur, 1981, for a recent restatement and proof of standard results), the population will converge to a stable population with a constant proportional age distribution. Births and the size of every age group will grow at some constant growth rate $g$. Expressed in discrete time, $B_{t}=B_{0}(1+g)^{t}$, and therefore $L_{i, t}=B_{t}(1+g)^{-i}$. Total population size at time $t$ will be $P_{t}=\sum_{0}^{\omega} L_{i, t} p_{i}=B_{t} \sum_{0}^{\omega}(1+g)^{-i} p_{i}$, where $\omega$ is the highest age in the population.

For demographic simplicity, assume all workers die at exactly age $\omega$, so that mortality can be ignored. Equivalently, assume that all workers survive from the age of entry into the labor force until some retirement age $\omega$. Mortality before and after working life can be ignored, with "births" referring to labor force entrants, and $g$ representing the labor force growth rate. Defining $\pi_{i}$ as the (constant) proportion of workers aged $i$ in the steady state, the effect of a fertility induced change in the population growth rate on this proportion is

$$
\frac{\partial \pi_{i}}{\partial g}=\frac{\pi_{i}}{(1+g)}(\bar{i}-i),
$$

where $\bar{i}=\sum_{i} \pi_{i} i$ is the mean age of the labor force. An increase in the population growth rate causes an increase in the steady state proportion of workers at all ages below the mean age and a decrease in the proportion of workers at all ages above the mean age. Assuming constant returns technology, the marginal products of workers are unaffected by total population size, and are therefore constant in the steady state, determined only by the relative sizes of age groups in a pure labor economy. If wages at each age are equal to marginal products, then total lifetime wages are given by $W=\sum_{i} F_{i}=\sum_{i} f_{i}$. The effect of the population growth rate on lifetime wages is therefore

$$
\begin{aligned}
\frac{\partial W}{\partial g} & =\sum_{i} \sum_{j} f_{i j} \frac{\partial \pi_{j}}{\partial g}=(1+g)^{-1} \sum_{i} \sum_{j} \pi_{j}(\bar{j}-j) f_{i j} \\
& =(1+g)^{-1} \sum_{i}\left[\bar{j} \sum_{j} \pi_{j} f_{i j}-\sum_{j} j \pi_{j} f_{i j}\right] .
\end{aligned}
$$

This result holds for any production function, as long as $g$ affects only the sizes of age groups and not the relative quantity of other productive factors. In the case of a pure labor economy with constant returns, the following proposition holds:

Proposition 2. In a pure labor economy with a concave constant returns production function $Y=F\left(L_{1}, L_{2}, \ldots, L_{\omega}\right)$, where $L_{i}=B_{t}(1+g)^{-i}$, with the labor force growing at a constant growth rate $g$, total lifetime wages $W=\sum_{i} F_{i}$ attain a global minimum when $g=0$. No finite growth rate exists that maximizes lifetime wages. 
To prove Proposition 2, note that in the case of a pure labor economy with constant returns, Euler's theorem requires that $\sum_{j} \pi_{j} f_{i j}=0$. This implies that

$$
\begin{aligned}
\frac{\partial W}{\partial g} & =-(1+g)^{-1} \sum_{i} \sum_{j} j \pi_{j} f_{i j}=-(1+g)^{-1} \sum_{j} j \pi_{j} \sum_{i} f_{i j} \\
& =-(1+g)^{-1} \sum_{j} j \pi_{j}\left[f_{j j}+\sum_{i \neq j} f_{i j}\right] .
\end{aligned}
$$

Under the assumption of a pure labor economy with constant returns, $\pi_{j} f_{j j}=-\sum_{i \neq j} \pi_{i} f_{i j}$, so (10) can be similified to

$$
\begin{aligned}
\frac{\partial W}{\partial g} & =(1+g)^{-1}\left[\sum_{j} j \sum_{i \neq j} \pi_{i} f_{i j}-\sum_{j} j \pi_{j} \sum_{i \neq j} f_{i j}\right] \\
& =(1+g)^{-1} \sum_{j} \sum_{i \neq j} j\left(\pi_{i}-\pi_{j}\right) f_{i j} .
\end{aligned}
$$

By (11) it is clear that $W(g)$ attains a critical point at $g=0$, since $\pi_{i}=\pi_{j} \forall(i, j)$ in a stationary population with no mortality before age $\omega$. (With mortality during the years workers are in the labor force a uniform age distribution could only exist with a negative population growth rate). To evaluate this critical point, it is useful to rewrite the expression. Note that $\sum_{j} \sum_{i \neq j} x_{i j}=\sum_{j} \sum_{i>j}\left(x_{i j}+x_{j i}\right)$. Since $f_{i j}=f_{j i}$, it follows that

$$
\begin{aligned}
\sum_{j} \sum_{i \neq j} j\left(\pi_{i}-\pi_{j}\right) f_{i j} & =\sum_{j} \sum_{i>j}\left[j\left(\pi_{i}-\pi_{j}\right) f_{j i}+i\left(\pi_{j}-\pi_{i}\right) f_{i j}\right] \\
& =\sum_{j} \sum_{i>j}\left(\pi_{i}-\pi_{j}\right)(j-i) f_{i j} .
\end{aligned}
$$

Substituting from (12), then, (11) can be rewritten as

$$
\frac{\partial W}{\partial g}=(1+g)^{-1} \sum_{j} \sum_{i>j}\left(\pi_{i}-\pi_{j}\right)(j-i) f_{i j}
$$

The second derivative is

$$
\begin{aligned}
\frac{\partial^{2} W}{\partial g^{2}}= & -(1+g)^{-2}(1+g) \frac{\partial W}{\partial g}+(1+g)^{-1} \sum_{j} \sum_{i>j}\left[(j-i) f_{i j}\left(\frac{\partial \pi_{i}}{\partial g}-\frac{\partial \pi_{j}}{\partial g}\right)\right] \\
& +(1+g)^{-1} \sum_{j} \sum_{i>j}\left[\left(\pi_{i}-\pi_{j}\right)(j-i)\left[f_{i j i} \frac{\partial \pi_{i}}{\partial g}-f_{i j j} \frac{\partial \pi_{j}}{\partial g}\right]\right] .
\end{aligned}
$$

At a uniform age distribution $\pi_{i}=\pi_{j}$, so the first term and last terms go to zero. The second term is unambiguously positive, since $\frac{\partial \pi_{i}}{\partial g}>\frac{\partial \pi_{j}}{\partial g} \forall i<j$. A uniform labor force age distribution thus gives the unambiguous global minimum lifetime wages for any constant returns production function in a pure labor economy. This establishes Proposition 2.

In a pure labor economy under constant returns the absolute size of the population has no effect on wages - only the relative sizes of age groups matter. If 
non-labor factors are introduced then it is necessary to explicitly model the effect of population growth on those factors. If the non-labor factors are fixed resources, such as land, then the relative supply of the factor must necessarily decrease with population size and there is no steady state level of output per worker. If the non-labor factor is capital then a formal model of capital accumulation is required. Assuming that a steady state capital-labor ratio exists, some model is required to analyze the effect of population growth on the capital-labor ratio. As pointed out above, this issue has been the principal focus of most previous literature on the economic effects of population growth. It is beyond the scope of this paper to offer any new approaches to the relationship between population growth and capital accumulation. One standard approach, the assumption that golden-rule savings is achieved in every steady state, will be used below. More generally, the effect of population growth on capital-labor ratios can simply be thought of as an additional term affecting lifetime wages in some unknown direction. If this term is strongly negative, as implied by most previous models, then it may dominate the tendency for higher rates of population growth to increase lifetime wages in a pure labor economy.

Assuming a steady state capital-labor ratio $k$ always exists, (9) continues to hold when a non-labor factor $K$ is introduced, but Euler's theorem now implies that $\sum_{j} \pi_{j} f_{i j}=-k f_{i k}$. Substituting into (9) and allowing an effect of $g$ on $k$, the result is

$$
\begin{aligned}
\frac{\partial W}{\partial g} & =(1+g)^{-1} \sum_{i} \bar{j} k f_{i k}-\sum_{i} \sum_{j} j \pi_{j} f_{i j}+\frac{\partial k}{\partial g} \sum_{i} f_{i k} \\
& =(1+g)^{-1}\left[\sum_{j} \sum_{i>j}\left(\pi_{i}-\pi_{j}\right)(j-i) f_{i j}+k\left(\sum_{i} f_{i k}(i-\bar{i})\right)\right]+\frac{\partial k}{\partial g} \sum_{i} f_{i k} .
\end{aligned}
$$

If it is assumed that $\partial k / \partial g=0$, and if $\partial W / \partial g=0$ at a uniform age distribution, then $\sum_{i} f_{i k}(i-\bar{i})=0$, since the first term in (15) has already been shown to be zero when $\pi_{i}=\pi_{j} \forall(i, j)$. If this is true, however, then it must be the case that $f_{i k}=f_{j k} \forall(i, k)$ when $\pi_{i}=\pi_{j}$. Even ignoring the final term in (15), there are no general restrictions on the third cross-partial derivatives to establish whether this critical point is a minimum or maximum, as discussed above in the case of two types of workers. If an increase in the population growth rate decreases the capital-labor ratio, as it does in a simple neoclassical growth model, then the last term further modifies the result, implying a negative effect of population growth on lifetime wages as long as $f_{i k}>0$ for all $i$.

\section{Effects of discounting lifetime wages}

It is straightforward to introduce a discount rate into the wage stream. Returning to the pure labor economy, if we redefine $W$ as discounted lifetime wages $W=$ $\sum_{i} F_{i}(1+r)^{-i}$, and analyze the effects of the population growth rate on $W$, the result in (11) generalizes to 


$$
\begin{aligned}
\frac{\partial W}{\partial g}= & (1+g)^{-1} \sum_{j} \sum_{i \neq j} j\left[\pi_{i}(1+r)^{-j}-\pi_{j}(1+r)^{-i}\right] f_{i j} \\
& -\frac{\partial r}{\partial g}(1+r)^{-1} \sum_{i} i w_{i}(1+r)^{-i} .
\end{aligned}
$$

The second term in (16) allows the possibility that the discount rate $r$ is itself a function of $g$. Assuming for the moment that $r$ is exogenous, making the second term in (16) equal to zero, the result implies that there will be a critical point at the growth rate which sets $\pi_{i}(1+r)^{-j}=\pi_{j}(1+r)^{-i}$ for all $(i, j)$. Continuing to abstract from mortality, this will occur when $g=r$, the population growth rate equals the discount rate. As in the case when $r=0$, it is easy to show that this critical point always gives the minimum discounted value of lifetime wages. For any exogenous discount rate $r$, discounted lifetime wages attain a global minimum when the population growth rate equals the discount rate. Evaluated at a point at which $g<r$ (e.g. $g=0.01$ and $r=0.10$ ), an increase in $g$ will decrease the discounted value of lifetime wages.

The result is more complicated if $r$ is a function of $g$. One important special case is when $r=g$ at all values of $g$, corresponding to Samuelson's (1958) "biological interest rate". This case has some intuitive appeal, since it captures the relationship between age structure and the possibilities for intergenerational borrowing. (See Willis 1982, for an excellent treatment of the relationship between age structure and intergenerational debt.) The value of earning high old-age wages to offset low young-age wages in a rapidly growing population depends on the ability to borrow against old-age wages. If the young can only borrow from the old, then the interest rate must increase as the population growth rate increases. In the case when $r=g$ at all values of $g$, the result in (16) can be rewritten by substituting $g$ for $r$, noting that $\partial r / \partial g=1$ and $(1+g)^{-i}=\pi_{i} \sum_{j}(1+g)^{-j}$ :

$$
\frac{\partial W}{\partial g}=\sum_{i}(1+g)^{-i-1}\left[\sum_{i} \pi_{i} \sum_{j} j \pi_{j} f_{i j}-\sum_{i} i \pi_{i} F_{i}\right]=-y \bar{i}_{w} \sum_{i}(1+g)^{-i-1}
$$

where $y$ is per worker output and $\bar{i}_{w}=\sum_{i} i \pi_{i} w_{i} / \sum_{i} \pi_{i} w_{i}$ is a weighted mean age in which each age is weighted by the proportion of total wages earned at that age. The result in (17) is unambiguously negative, implying that if the discount rate $r$ is always equal to the population growth rate $g$, then increases in the growth rate must reduce the discounted value of lifetime wages. Although lifetime wages will always increase with increases in the population growth rate, in accordance with Proposition 2, they can never increase at the same rate as the population growth rate itself. In this biological interest rate case, then, the discounted value of lifetime wages is maximized at the most negative feasible population growth rate.

Although discounting lifetime wages may be appropriate, especially in the biological interest rate regime, the value of discounted lifetime wages is still not necessarily an appropriate objective function for the representative worker. The discounted cost of any given lifetime consumption stream also declines as the population growth rate increases. This means that a decline in discounted lifetime wages does not necessarily imply a decline in lifetime utility. The following sec- 
tions clarify this point by incorporating the effects of age structure on wages with the effects on lifetime consumption possibilities.

\section{Age structure and social budget constraints with overlapping generations}

The role of population growth and age structure in overlapping generations models has been clarified by Arthur and McNicoll $(1977,1978)$ and Lee $(1980)$. Extending results from formal demography, these papers combine intergenerational transfer consumption-loan effects with capital dilution effects in models with continuous age distributions. The same approach can be used to provide additional insights into the results proven above regarding the effects of age structure on age-specific labor productivity.

Before introducing effects of age structure on age-specific productivity, it is instructive to examine comparative steady state results for models in which workers at different ages are perfect substitutes. Consider a continuous time version of the stable population analyzed above, with constant age-specific fertility and mortality implying a constant exponential growth rate $g$, with $B_{t}=B_{0} \mathrm{e}^{-g t}$, and $N_{a, t}=B_{t} \mathrm{e}^{-g a}$. Total population size at time $t$ is $P_{t}=\int_{0}^{\omega} N_{a, t} p_{a} \mathrm{~d} a=$ $B_{t} \int_{0}^{\omega} \mathrm{e}^{-g a} p_{a} \mathrm{~d} a$, where $\omega$ is highest age in the population. Assume that consumption at age $a$ is given by the schedule $c_{a}$. We can assume that age-specific consumption is chosen to maximize lifetime utility, as in Arthur and McNicoll (1977), although as Lee (1980) shows, the principal insights of the comparative steady state results come from simply differentiating the social budget constraint to find the change in lifetime consumption possibilities. Details of how age-specific consumption is altered in response to a change in population growth add little additional information. Labor supply is given by an analogous function of age $l_{a}$, and period $t$ production is described by a concave constant returns production function $F\left(K_{t}, L_{t}\right)$, where $L_{t}$ is the effective labor force at time $t$. Total labor $L_{t}$ is a linear aggregation of the number of workers at each age, $L_{t}=B_{t} \int_{0}^{\omega} \mathrm{e}^{-g a} p_{a} l_{a} \mathrm{~d} a$. In most pure consumption loan models, workers at each age receive exogenous endowments which are unaffected by population growth (see, for example, Samuelson 1958, and Willis 1982). In models with capital, wages are affected by capital-labor ratios, and may also be affected by age-specific weights such as the $l_{a}$ terms used here. Arthur and McNicoll (1978) and Lee (1980) differ somewhat in their interpretation of the weights $l_{a}$ in the definition of $L_{t}$. Arthur and McNicoll define them as age-specific labor force participation rates, while Lee includes differences in productivity as reflected in wage differentials. These productivity differences are exogenous with respect to age structure, however, and therefore implicitly assume that workers of all ages are perfect substitutes.

The social budget constraint at time $t$ is $F\left(K_{t}, L_{t}\right)=C_{t}+\dot{K}_{t}$, where $C$ is total consumption and $\dot{K}$ is the time derivative of the capital stock. Generalizing Solow's growth model without age structure, this age-strucuted economy has an economic-demographic steady state growth path with a constant proportional age distribution, a constant capital-labor ratio $k$ and constant age-specific consumption levels $c_{a}$. In the steady state $\dot{K}_{t}=g K$, so normalizing by $B_{t}$ the social budget constraint can be rewritten as 


$$
[f(k)-g k] \int_{0}^{\omega} \mathrm{e}^{-g a} p_{a} l_{a} \mathrm{~d} a=\int_{0}^{\omega} \mathrm{e}^{-g a} p_{a} c_{a} \mathrm{~d} a .
$$

Following Arthur and McNicoll $(1977,1978)$, the simplest case is to assume that capital is accumulated by a golden-rule steady state savings rate, implying that $f_{k}=g$. The golden rule assumption implies that a new optimal savings rate which maximizes per capita consumption is chosen whenever the population growth rate changes. Drop the $t$ subscripts to denote the time invariant normalized total quantities $L=L_{t} / B_{t}, C=C_{t} / B_{t}$, and define the time invariant proportions $\pi_{a}=\mathrm{e}^{-g a} p_{a} / \int_{0}^{\omega} \mathrm{e}^{-g a} p_{a} \mathrm{~d} a, \pi_{a}^{c}=\mathrm{e}^{-g a} p_{a} c_{a} / \int_{0}^{\omega} \mathrm{e}^{-g a} p_{a} c_{a} \mathrm{~d} a$, and $\pi_{a}^{l}=$ $\mathrm{e}^{-g a} p_{a} l_{a} / \int_{0}^{\omega} \mathrm{e}^{-g a} p_{a} l_{a} \mathrm{~d} a$, where $\pi_{a}$ is the proportion of the population that is age $a, \pi_{a}^{c}$ is the proportion of total consumption consumed by persons aged $a$, and $\pi_{a}^{l}$ is the proportion of the labor force that is age $a$. The fundamental comparative steady state result is derived by differentiating the social budget constraint (18) with respect to $g$ (implying an increase in fertility with mortality held constant), imposing the golden rule savings condition that $f_{k}=g$, and noting that $(y-g k) L=C$ by the budget constraint (see Arthur and McNicoll 1978: 244, and Lee 1980: 1145):

$$
-k L+C \int_{0}^{\omega} \pi_{a}^{l} \frac{\partial \ln l_{a}}{\partial g} \mathrm{~d} a-C \int_{0}^{\omega} a \pi_{a}^{l} \mathrm{~d} a=C \int_{0}^{\omega} \pi_{a}^{c} \frac{\partial \ln c_{a}}{\partial g} \mathrm{~d} a-C \int_{0}^{\omega} a \pi_{a}^{c} \mathrm{~d} a .
$$

This result can be simplified to

$$
\int_{0}^{\omega} \pi_{a}^{c} \frac{\partial \ln c_{a}}{\partial g} \mathrm{~d} a=\bar{a}_{c}-\bar{a}_{l}-\frac{k}{c}+\int_{0}^{\omega} \pi_{a}^{l} \frac{\partial \ln l_{a}}{\partial g} \mathrm{~d} a .
$$

Two weighted mean ages appear in (20): $\bar{a}_{c}$ is the mean age of the cross-section population when each age is weighted by its share in total consumption; $\bar{a}_{l}$ is simply the mean age of the labor force. It is a standard result in mathematical demography that the effects of changes in the population growth rate on crosssection population aggregates are described by mean ages and weighted mean ages (see Coale 1972; Keyfitz 1977; Preston 1982). Lam (1984) generalizes the result for population means to higher moments of distributions of population characteristics. The interpretation of (20) can be seen with a simple special case. If all changes in consumption and labor supply implied by (20) are absorbed by constant proportional adjustments at each age, so that $\partial \ln c_{a} / \partial g=\gamma$ for all $a$ and $\partial \ln l_{a} / \partial g=\lambda$ for all $a$, then (20) reduces to

$$
\gamma=\left(\bar{a}_{c}-\bar{a}_{l}\right)-\frac{k}{c}+\lambda
$$

The proportional change in consumption at each age in response to an increase in the steady state population growth rate will be equal to the "average age of consumption" minus the "average age of production" minus the capital-consumption ratio plus the proportional change in labor effort at each age. If consumption occurs on average at older ages than production, as in Samuelson's consumption loan model in which there is a period of work followed by a period of retirement, then the average age terms imply a positive intergenerational transfer effect. If 
childhood consumption is included in the model, then the sign of the intergenerational transfer effect is ambiguous, and Arthur and McNicoll (1978) suggest that it can easily be negative. Whatever the sign of this effect, there will be an unambiguously negative capital dilution effect of minus the capital consumption ratio. Arthur and McNicoll (1978) and Lee (1980) suggest that this effect is likely to swamp any plausible positive intergenerational transfer effect. Finally, population growth may lead to increases or decreases in age-specific labor force participation rates.

The simple neoclassical growth model and consumption loan model can be seen to be special cases of (21). In a growth model with no age structure, such as Solow (1956), consumption and production always take place at the same age, so that the intergenerational transfer effect disappears. In the absence of an effect of population growth on labor effort, this leaves the standard capital dilution effect of population growth for golden rule steady states. In a simple consumption loan model there is no capital, leaving only the intergenerational transfer effect.

\section{Age structure and labor productivity}

The continuous age structure models of Arthur and McNicoll and Lee, like the related overlapping generations models of Samuelson (1958, 1976), Deardorff (1976), and Willis (1982), make very simple assumptions about age-specific labor productivity. In the pure consumption loan model of Samuelson (1958), life cycle wage profiles are constant, and are simply modeled as age-specific endowments. The advantages of higher rates of population growth result from the relationship between age structure and the ability to make intergenerational consumption loans, not from any effect of age structure on age-specific productivity. When capital is introduced in Samuelson's later work $(1975,1976)$ and in the work of Arthur and McNicoll $(1977,1978)$ and Lee $(1980)$, wages are affected, but labor is homogeneous except for exogenous variations across age in labor supply or effort. In other words, labor is simply a linear aggregation of workers of all ages. Wages at all ages rise and fall together as the capital-labor ratio responds to varying population growth rates. Wages vary across ages only because of the exogenous difference in age-specific labor supply or effort which are built into the models.

Given the theoretical results presented above and the empirical evidence on the effects of relative age group size on age-specific wages, it is instructive to consider how models of the effects of population growth are affected by explicitly modeling age-specific productivity as a function of the relative sizes of all age groups. A more realistic model, for example, will have wages of young workers moving in the opposite direction from wages of old workers in response to an increase in the population growth rate.

More realistically, then, modify the model above to let output depend explicitly on the number of workers at each age $Y_{t}=F\left(K_{t}, L_{0, t}, \ldots, L_{a, t}, \ldots, L_{\omega, t}\right)$, where $L_{a, t}$ is the number of workers aged $a$ in period $t$. The model can be generalized in this way and yet kept quite tractable by continuing to assume that the production function is constant returns to scale. 
It is still true that the budget constraint $Y_{t}=C_{t}-\dot{K}_{t}$ must be satisfied in every period, and that $\dot{K}=g K$ in the steady state. Continue to let $l_{a}$ represent the labor force participation rate of workers aged $a$. Normalizing by labor force size $L_{t}=B_{t} \int_{0}^{\omega} \mathrm{e}^{-g a} p_{a} l_{a} \mathrm{~d} a$, the linearly homogeneous production function can be normalized to $y=Y_{t} / L_{t}=\left(k, \pi_{0}^{l}, \ldots, \pi_{a}^{l}, \ldots, \pi_{\omega}^{l}\right)$, where $\pi_{a}^{l}$, as defined above, is the proportion of the total labor force made up of workers aged $a$. The only modification to the budget constraint in (18), then, is to generalize per worker output, implying that

$$
\left[f\left(k, \pi_{0}^{l}, \ldots, \pi_{a}^{l}, \ldots, \pi_{\omega}^{l}\right)-g k\right] \int_{0}^{\omega} \mathrm{e}^{-g a} p_{a} l_{a} \mathrm{~d} a=\int_{0}^{\omega} \mathrm{e}^{-g a} p_{a} c_{a} \mathrm{~d} a .
$$

Let $w_{a}$ denote the marginal product of workers aged $a$. We can interpret $w_{a}$ as the competitive wage, although a model of wage determination is not necessary for the results which follow. Differentiating (22) with respect to $g$,

$$
\begin{aligned}
L\left[\frac{\partial k}{\partial g}\left(f_{k}-g\right)\right. & \left.-k+\int_{0}^{\omega} w_{a} \frac{\partial \pi_{a}^{l}}{\partial g} \mathrm{~d} a\right] \\
& +(y-g k)\left[\int_{0}^{\omega} \mathrm{e}^{-g a} p_{a} \frac{\partial l_{a}}{\partial g} \mathrm{~d} a-\int_{0}^{\omega} a \mathrm{e}^{-g a} p_{a} l_{a} \mathrm{~d} a\right] \\
= & \int_{0}^{\omega} \mathrm{e}^{-g a} p_{a} \frac{\partial c_{a}}{\partial g} \mathrm{~d} a-\int_{0}^{\omega} a \mathrm{e}^{-g a} p_{a} c_{a} \mathrm{~d} a .
\end{aligned}
$$

Imposing the golden rule savings condition that $f_{k}=g$ and noting that $(y-g k)$ $L=C$ by the budget constraint, (23) can be rewritten as

$$
L \int_{0}^{\omega} w_{a} \frac{\partial \pi_{a}^{l}}{\partial g} \mathrm{~d} a-k L+C \int_{0}^{\omega} \pi_{a}^{l} \frac{\partial \ln l_{a}}{\partial g} \mathrm{~d} a-C \bar{a}_{l}=C \int_{0}^{\omega} \pi_{a}^{c} \frac{\partial \ln c_{a}}{\partial g} \mathrm{~d} a-C \bar{a}_{c} .
$$

The result looks identical to the result for the case of perfect substitutability in (19), except for the first term involving the marginal product of each age worker. In the previous model all workers have the same marginal product, $f_{l}=y-f_{k} k$. If this assumption is imposed and substituted into (24), the first term becomes $L\left(y-f_{k} k\right) \int_{0}^{\omega} \frac{\partial \pi_{a}^{l}}{\partial g} \mathrm{~d} a$. The term vanishes, since $\int_{0}^{\omega} \frac{\partial \pi_{a}^{l}}{\partial g} \mathrm{~d} a=0$, reducing the model to the models of Arthur and McNicoll or Lee in which workers of all ages are perfect substitutes.

In the more general case in which workers of different ages are not perfect substitutes, it is straightforward to show that the effect of the population growth rate on the proportion of the labor force at a given age $\hat{a}$ is

$$
\frac{\partial \pi_{\hat{a}}^{l}}{\partial g}=\pi_{\hat{a}}^{l}\left[\bar{a}_{l}-\hat{a}+\frac{\partial \ln l_{\hat{a}}}{\partial g}-\int_{0}^{\omega} \pi_{a}^{l} \frac{\partial \ln l_{a}}{\partial g} \mathrm{~d} a\right] .
$$

This implies that 


$$
\begin{aligned}
\int_{0}^{\omega} w_{a} \frac{\partial \pi_{a}^{l}}{\partial g} \mathrm{~d} a & =\bar{w}\left[\bar{a}_{l}-\int_{0}^{\omega} \pi_{a}^{l} \frac{\partial \ln l_{a}}{\partial g} \mathrm{~d} a\right]+\int_{0}^{\omega} \pi_{a}^{l} w_{a} \frac{\partial \ln l_{a}}{\partial g} \mathrm{~d} a-\int_{0}^{\omega} a \pi_{a}^{l} w_{a} \mathrm{~d} a \\
& =\bar{w}\left[\bar{a}_{l}-\bar{a}_{w}+\int_{0}^{\omega} \pi_{a}^{w} \frac{\partial \ln l_{a}}{\partial g} \mathrm{~d} a-\int_{0}^{\omega} \pi_{a}^{l} \frac{\partial \ln l_{a}}{\partial g} \mathrm{~d} a\right],
\end{aligned}
$$

where $\pi_{a}^{w}=\mathrm{e}^{-g a} p_{a} l_{a} w_{a} / \int_{0}^{\omega} \mathrm{e}^{-g a} p_{a} l_{a} w_{a} \mathrm{~d} a$, the proportion of total wages earned by workers age $a, \bar{w}=\int_{0}^{\omega} \pi_{a}^{w} w_{a} \mathrm{~d} a$, the mean wage in the working population, and $\bar{a}_{w}=\int_{0}^{\omega} a \pi_{a}^{l} w_{a} \mathrm{~d} a$, the weighted mean age of the labor force when each age is weighted by the proportion of total wages earned at that age.

Substituting (25) into (24), imposing the requirement that $\bar{w} L=C$ in golden rule steady states, and rearranging terms, the effect of a fertility induced change in the population growth rate on steady state lifetime consumption can be summarized as

$$
\int_{0}^{\omega} \pi_{a}^{c} \frac{\partial \ln c_{a}}{\partial g} \mathrm{~d} a=\bar{a}_{c}-\bar{a}_{w}-\frac{k}{c}+\int_{0}^{\omega} \pi_{a}^{w} \frac{\partial \ln l_{a}}{\partial g} \mathrm{~d} a .
$$

The only difference between the comparative steady state result in (26) and the result in (20) is that the mean age of the labor force and the integral of changes in labor supply are weighted by age-specific wages in the new result. The similarity is at first surprising, since (26) describes the effect of population growth for any general constant returns production function with every age worker considered as a separate factor of production. No assumption has been made about the elasticity of substitution between workers of different ages. The result is completely general in this respect, allowing, for example, for workers close in age to be substitutes while workers farther apart in age are complements, or alternatively allowing workers of all ages to be either substitutes or complements.

How can it be that (26) summarizes the effects of population growth on per capita lifetime consumption possibilities for any constant returns production function, without any specification of the substitutability of workers of different ages? Surely there is some basis to the intuition that the effects of a change in age structure in an economy where workers of all ages are perfect substitutes will be very different than the effects in an economy where there is very limited substitutability across ages. The answer is that all information about the elasticities of substitution across age groups of workers and between labor and capital is already captured in the weighted mean ages in (26). The result is less surprising when it is recalled that the result in (20) describes the effects of population growth for any constant returns function of capital and labor without any specification of the elasticity of substitution between capital and labor. In both cases it is not that the elasticity of substitution does not matter. It is rather that its effects are entirely captured in the mean age terms that summarize the comparative steady state result.

\section{Analogies to Samuelson consumption-loan economies}

The results derived above imply that higher rates of population growth may lead to increased lifetime wages and increased lifetime consumption for a represen- 
tative worker. Such effects are similar to the potential windfalls from more rapid population growth in a pure consumption loan economy with intergenerational transfers, as originally modeled by Samuelson (1958). In Samuelson's overlapping generations economy, the lifetime incomes of workers are constant, modeled as exogenous age-specific endowments. Changes in age structure have no effect on these age-specific wages. The lifetime utility of workers is affected by changes in age structure, not because of changes in income profiles, but because of changes in the potential for intergenerational borrowing and lending. If a pay-as-you-go social security system is maintained, for example, higher rates of population growth lead to higher lifetime utility because of the increased ratio of contributors to dependents.

The results of this paper depend on a different mechanism through which age structure affects the lifetime welfare of a representative worker. Exogenous agespecific endowments are replaced with age-specific wages, where the wages are marginal products from a concave constant returns production function. For a pure labor economy, the closest analog to Samuelson's pure consumption loan economy, the paper proves that lifetime wages attain a global minimum when there is a uniform age distribution. Persistent positive or negative population growth rates, assuming they can be maintained, always generate higher lifetime wages than those in a stationary population.

To see the contrast between the effects of age structure modeled here and the effects resulting from intergenerational consumption loans, note from Proposition 2 that lifetime wages are unambiguously increased when the age structure deviates from uniformity in either direction, i.e. when the population growth rate becomes either positive or negative. The benefits from altering the age structure through intergenerational transfers, by contrast, must always be asymmetric. If a positive growth rate raises lifetime consumption possibilities compared to stationarity through intergenerational transfer effects, then a negative growth rate must necessarily decrease lifetime consumption possibilities. The difference results from the fact that intergenerational transfers play no role in Proposition 2. Lifetime wages are increased by skewing the age distribution in either direction as a necessary implication of concavity and linear homogeneity of the production function. The assumption that workers of different ages are perfect substitutes, an assumption made either explicitly or implicitly in the models of Samuelson, Arthur and McNicoll, Lee, and Willis represents the limiting case of Proposition 2 in which lifetime wages will be independent of age structure. In such a case intergenerational transfers will be the only mechanism through which age structure affects lifetime consumption in a pure labor economy.

The results derived above imply that even in the absence of intergenerational transfers, fertility-induced increases in the population growth rate would increase the utility of workers if lifetime utility were an increasing function of lifetime wages. Skewed age distributions may imply lower per capita wages in every period, but will always imply higher lifetime wages in the case of a pure labor economy. The surprising result that lifetime wages tend to decrease as the age structure moves closer to uniformity does not appear to have been previously recognized in theoretical analysis of age-earnings profiles or in models of the economic effects of population growth. 


\section{Conclusions}

Empirical evidence on the effects of cohort size on age-specific wages suggests that there will be significant interactions between population growth rates and life cycle wage profiles both in the short run and in long run demographic steady states. This paper begins with the question of whether there will exist a steadystate age distribution that maximizes the lifetime wages of a representative worker when workers of different ages are imperfect substitutes. The paper proves the surprising result that in a pure labor economy with any constant returns to scale technology there is no finite population growth rate that maximizes lifetime wages. A uniform age distribution (corresponding to zero population growth in the absence of mortality) is shown to produce the minimum total value of lifetime wages in a pure labor economy, a result which holds for any constant returns technology, independent of the relative productivity of different ages or the elasticities of substitution across age groups. Total lifetime wages increase in response to movements away from stationarity in either a positive or negative direction, with the increase unbounded in both directions.

If lifetime wages are discounted at some constant rate, the result generalizes to the condition that discounted lifetime wages attain a global minimum when the population growth rate equals the discount rate. This result may not hold if the discount rate is itself a function of the population growth rate. In the special case in which the discount rate is always equal to the population growth rate, corresponding to Samuelson's "biological interest rate", discounted lifetime wages are shown to unambiguously fall with increases in the population growth rate.

The presence of non-labor factors complicates, but does not necessarily reverse, the tendency for uniformity in the age distribution to minimize lifetime wages. If the population growth rate directly affects capital-labor ratios, as argued in most previous models of the economic effects of population growth, capital dilution effects may overcome the effects of population growth on lifetime wage profiles.

Lifetime wages, discounted or not, cannot describe lifetime utility without consideration of lifetime consumption profiles. In order to capture these effects and to consider the role of capital accumulation, the paper incorporates the effects of age structure on age-specific labor productivity into models which analyze cross-section social budget constraints in golden rule economicdemographic steady states. It is proven that previous comparative steady state results based on the assumption that workers of different ages are perfect substitutes continue to hold for any assumption about the elasticity of substitution between workers of different ages, providing the "mean ages" which determine the result are appropriately defined. The surprising robustness of the previous results occurs not because the elasticities of substitution between workers of different ages do not matter, but because their effects are captured in the "wageweighted mean age" of the labor force.

The results demonstrate a number of important and previously unrecognized effects of age structure on life-cycle wage profiles. The results should not necessarily be interpreted as providing new ammunition for debates over population policy. Issues of population policy are better analyzed by looking directly at 
the optimality of private fertility decisions, as in Nerlove et al. (1987) and Willis (1987). The effects of age structure on lifetime wages established in this paper do provide useful insights into the changes in wage profiles that will be observed as populations move closer to or farther away from uniform age distributions, and fill an important gap in previous models of the economic effects of changing age structure.

\section{References}

Arthur WB (1981) The ergodic theorems of demography: a simple proof. Demography 19:439-445 Arthur WB, McNicoll G (1977) Optimal time-paths with age dependence: a theory of population policy. Rev Econ Studies 44:111-123

Arthur WB, McNicoll G (1978) Samuelson, population and intergenerational transfers. Int Econ Rev 19:241-246

Berger MC (1985) The effect of cohort size on earnings growth: a reexamination of the evidence. $J$ Polit Econ 93:561-573

Berger MC (1989) Demographic cycles, cohort size, and earnings. Demography 26:311-321

Bloom DE, Freeman RB, Korenman SD (1988) The labour-market consequences of generational crowding. Eur J Popul 3:131-176

Coale AJ (1972) The growth and structure of human populations: a mathematical investigation. Princeton University Press, Princeton

Connelly R (1986) A framework for analyzing the impact of cohort size on education and labor earnings. J Hum Resources 21:543-562

Deardorff A (1976) The optimum growth rate for population: comment. Int Econ Rev 17:510-515

Dooley MD, Gottschalk P (1984) Earnings inequality among males in the U.S.: trends and the effect of labor force growth. J Polit Econ 92:59-89

Easterlin RA (1978) What will 1984 be like? Socioeconomic implications of recent twists in age structure. Demography 15:397-432

Falaris EM, Peters HE (1988) Fertility, female labor supply, and the demographic cycle. Presented at IUSSP Seminar on The Family, The Market, and The State in Aging Societies, Sendai, Japan

Freeman R (1979) The effect of demographic factors on age-earnings profiles. J Hum Resources $14: 289-318$

Keyfitz N (1977) Applied mathematical demography. Wiley and Sons, New York

Lam D (1984) The variance of population characteristics in stable populations, with applications to the distribution of income. Popul Studies 38:117-127

Lee R (1980) Age structure, intergenerational transfers, and economic growth. Rev Econ $31: 1129-1156$

Lee R, Lapkoff S (1988) Intergenerational flows of time and goods: consequences of slowing population growth. J Polit Econ 96:618-651

Nerlove M, Razin A, Sadka E (1987) Household and economy: welfare economics of endogenous fertility. Academic Press, Boston

Preston S (1982) Relations between individual life cycles and population characteristics. Am Sociol Rev 47:253-264

Samuelson P (1958) An exact consumption-loan model of interest with or without the social contrivance of money. J Polit Econ 66:467-482

Samuelson P (1975) The optimum growth rate for population. Int Econ Rev 16:531-538

Samuelson P (1976) The optimum growth rate for population: agreement and evaluations. Int Econ $\operatorname{Rev} 17: 516-525$

Solow R (1956) A contribution to the theory of economic growth. Q J Econ 79:65-94

Stapleton DS, Young DY (1984) The effects of demographic change on the distribution of wages, 1967-1990. J Hum Resources 19:175-201

Stapleton DS, Young DY (1988) Educational attainment and cohort size. J Labor Econ 6:330-361

Welch $F$ (1979) Effects of cohort size on earnings: the baby boom babies' financial bust. J Polit Econ 87:S65-S 97

Willis R (1982) The direction of intergenerational transfers and demographic transition: the Caldwell hypothesis reexamined. Popul Dev Rev 8(Suppl):207-234

Willis R (1987) Externalities and population. In: Gale Johnson D, Lee RD (eds) Population growth and economic development: Issues and evidence. University of Wisconsin Press, Madison, pp 661-702 